\title{
LA RESPONSABILIDAD SOCIAL CORPORATIVA Y LA COMPATIBILIDAD ELECTROMAGNÉTICA EN UN CONTEXTO INTERNACIONAL
}

CORPORATE SOCIAL RESPONSABILITY AND ELETROMAGNETIC COMPABILITY IN AN INTERNATIONAL CONTEXT

\author{
GREGORIO ESCALERA IZQUIERDO \\ Doctor en Ciencias Económicas y Empresariales - Universidad Nacional \\ de Educación a Distancia (Uned). \\ Profesor de la Facultad Ciencias Económicas y Empresariales - Departamento \\ de Organización de Empresas. \\ Edificio de la Escuela Técnica Superior c/ Juan del Rosal, 16. Despacho 422 \\ 28040 - Madrid - España \\ E-mail: gescalera@cee.uned.es
}

\section{CARIMEN CALDERÓN PATIER}

Doctora en Ciencias Económicas y Empresariales Universidad San Pablo (CEU).

Profesora de la Facultad de Ciencias Económicas y Empresariales - Departamento de Economía General, c/ Julian Romea, 20 28003 - Madrid - España

E-mail: calder@ceu.es 


\section{RESUMEN}

El presente trabajo estudia los criterios de certificación internacionales de calidad referidos a la Compatibilidad Electromagnética (CEM), dirigidos a la protección de los sistemas de comunicación. Serán analizados los tres mercados de referencia, el europeo, norteamericano y japonés, que nos permitirán conocer el estadio actual de regulación, así como las posibles tendencias futuras de las diferentes áreas y zonas de producción en un contexto de globalización internacional.

\section{PALABRAS CLAVE}

Responsabilidad Social Corporativa; Compatibilidad electromagnética; Marcado CE, Marcado FCC; Marcado PSE.

\section{ABSTRACT}

The present work studies the international certification approaches of quality referred to the Electromagnetic Compatibility (CEM), aimed to the protection of the communication systems. Three reference markets will be analyzed, the European, North American and Japanese, that will allow us to know the current status of regulation, as well as the possible future tendencies of the different areas and production regions in a context of international globalization.

\section{INTRODUCCIÓN}

La definición que una sociedad o mercado tenga de la Responsabilidad Social Corporativa (en adelante, RSC) determina el grado o nivel de regulación del mismo, así como los posibles sistemas de certificación de la calidad de sus 
- LA RESPONSABILIDAD SOCIAL CORPORATIVA Y LA COMPATIBILIDAD ELETROMAGNÉTICA... •

productos. Se hace, por lo tanto, necesaria, antes de abordar el análisis de dichos criterios, estudiar la interpretación que realizan de la misma.

Una primera aproximación a RSC debería partir de los tres elementos que configuran la misma, esto es: las propias empresas, la sociedad en la que están inmersas y las personas que de alguna manera están vinculadas a dichas empresas.

Responsabilidad significa, para algunos, solidaridad, en interés por aquellos que se ven afectados por las consecuencias de nuestras acciones; de ahí derivaría una obligación básica y sin reciprocidad hacia el futuro. En este sentido, la responsabilidad atañe a la obligación de justificar todo acto o decisión en función de normas morales y de valores éticos.

Cuando este concepto lo aplicamos a la empresa, la responsabilidad social oscila entre quedar reducida a la obtención de un beneficio (el máximo posible) para los accionistas y la extensión de la responsabilidad a las relaciones y a la forma en que aquella influye sobre las decisiones de la organización y afecta a todos los interlocutores que tienen una clara relación de interés con la empresa. El debate estaría centrado en la finalidad de la empresa y su papel: si su objetivo único consiste en enriquecer a los accionistas, o puede, por el contrario, ser definido desde un punto de vista ético.

Si entendemos la RSC como un problema ético, hay que señalar que éstos aparecen siempre que las decisiones tomadas libremente por un individuo - y, en su caso, ejecutadas, bien directamente o bien a través de una organización como pudiera ser una empresa- tienen un impacto positivo o negativo sobre los demás. En este sentido las decisiones que se adoptan en las empresas, al ser tomadas por individuos y también al afectar a colectividades mucho más amplias de lo que es el ámbito interno de las organizaciones (sociedad en general, proveedores, clientes, etc.), no pueden ser entendidas como meras cuestiones de carácter analítico, pues siempre harán referencia a valores.

El objetivo del presente trabajo es contrastar si la interpretación que una economía otorga a la Responsabilidad Social Corporativa (RSC) influye o determina, no sólo el nivel de regulación del mercado, sino y más importante, los sistemas de certificación de la calidad de los productos que actualmente se producen, distribuyen y comercializan en todas las economías modernas. Para ello, se ha seleccionado un sector, pionero y altamente globalizado, como es el sector de productos electromagnéticos, y se tratará de analizar si los criterios actualmente vigentes para la certificación de la calidad de los mismos son homogéneos a nivel internacional, o bien por el contrario éstos vienen determinados por la interpretación particular que cada sociedad otorgue a la RSC.

En esta línea, el artículo se divide en tres grandes bloques. En primer lugar, se define el concepto de RSC en cada uno de los ámbitos objeto de estudio; Unión 
Europea, USA y Japón, necesario e imprescindible para comprender e enmarcar adecuadamentelas normas de calidad vigentes en cada uno de ellos. A continuación, nos centramos en la regulación de la Compatibilidad Electromagnética y su instrumentación en esos escenarios previamente definidos, y para finalizar, se realiza un análisis comparativo de las tres áreas a modo de conclusiones.

\section{RESPONSABILIDAD SOCIAL CORPORATIVA EN UN ENTORNO GLOBALIZADO}

La ética empresarial o ética en los negocios depende del marco económico, político, social, histórico y geográfico en el que nos situemos; así, cabe hablar de varios enfoques diferentes, según nos encontremos en Estados Unidos, en Japón o en Europa.

La visión americana se concreta en declaraciones y códigos por parte de las empresas acerca de sus posiciones éticas. Más del 90\% de las grandes corporaciones americanas tienen una política ética formalizada. Las empresas americanas fueron las primeras en iniciar los códigos éticos, como también lo fueron en hablar de responsabilidad social. La vía en que los americanos abordan los problemas éticos en las empresas deja traslucir una lógica utilitarista. La ética de los negocios, en este sentido, no pretende servir de ideal, sino ser un medio para conseguir unos determinados objetivos. La finalidad es la búsqueda de una mejor imagen y una mayor rentabilidad de las corporaciones, integradas en una concurrencia mundial sumamente agresiva.

Los americanos han materializado en leyes buena parte de sus ideas acerca del comportamiento de las corporaciones. En este sentido es difícil pensar en términos de ética en Estados Unidos fuera del marco legislativo; la manera más común y la más fácil para las empresas de responder a sus responsabilidades éticas es formular un código ético.

La cultura japonesa, por el contrario, lleva a las organizaciones de este país a una ética que pudiéramos denominar comunitaria: la ética es, para las empresas en el mundo nipón un elemento de identificación social y de sentimiento de pertenencia a un grupo, y en este sentido, los principios éticos se consideran como un fundamento y una premisa básica para el éxito.

La cultura económica europea, sumida en fuertes procesos de unificación económica y política e incluso social (marcada por la filosofía del Estado del Bienestar), presenta un enfoque, frente a estos problemas, notablemente diferente, y entre los diferentes países, los europeos son reticentes a abordar 
públicamente sus problemas de naturaleza ética por temor a exponerse a la crítica, por lo que a menudo utilizan medios indirectos para expresar responsabilidades éticas (legislación, negociaciones con los interlocutores sociales, etc.). En otro orden de cosas, se observa cómo en aquellos países cuyas legislaciones confieren más poder a gerentes frente a accionistas es donde se ve más clara la necesidad de exigencias de RSC y de códigos de buen gobierno.

Con independencia de los tres enfoques mencionados, anglosajón, asiático y europeo, parece que existe un cierto consenso en cuanto a la definición de la RSC dependiendo de la perspectiva desde la cual se analice. Y así podríamos diferenciar cuatro grandes dimensiones de la misma, todas ellas no excluyentes y complementarias entre si:

- Enunciado de valores y creencias: sobre la RSC puede ser entendida como una reflexión de naturaleza axiológica, esto es: valores como integridad, honestidad, equidad, lealtad y solidaridad pasan a incorporarse al acervo de las corporaciones y, generalmente, a formalizarse en documentos que explicitan la visión de si mismas y de la misión que tienen dichas corporaciones, concretando dichas declaraciones de principios o en códigos éticos.

- Credo o filosofía de gestión consiste en entender la RSC como una traslación de las normas deontológicas dadas a sí mismos por los integrantes de una determinada profesión, en este caso los directivos de empresas, que se traduce en una voluntad de adhesión de la empresa y de sus directivos a dichos principios

- El proyecto colectivo de la empresa sería la que considera la RSC como una reflexión teológica y ontológica, esto es, como una intención y explicitación de objetivos y de adhesión a las finalidades del entorno por parte de la organización y como una voluntad de reflexionar sobre su propia naturaleza.

- El código ético sería entenderla desde una óptica psicológica. Se trataría de entender a los miembros de la empresa como parte importante de la organización, y los códigos éticos servirían de nexo de unión entre los miembros que componen la organización y los fines de esta.

\section{LA COMPATIBILIDAD ELECTROMAGNÉTICA}

Para muchos el concepto de Compatibilidad Electromagnética (en adelante CEM), puede resultar relativamente novedoso. Sin embargo, su estudio ha ocupado a varias agencias desde los tiempos de la Segunda Guerra Mundial. Entre 
otras, diversos organismos europeos han sido fundamentales en la definición de normativas y recomendaciones para la fabricación y comercialización de productos electromagnéticos. En este sentido se analizan las directivas de la Unión Europea, normativa de Estados Unidos y normativa japonesa que marcan los criterios de certificación de calidad en lo referente a CEM. En este trabajo se relacionan estas directivas, buscando puntos de referencia comunes, diferencias relevantes, e incorporando otras normativas internacionales de calidad en el ámbito de la Compatibilidad Electromagnética. Para evaluar la tendencia o no a la uniformidad de la norma y su posible futura tendencia.

En la sociedad actual, debido a la proliferación global de equipos electrónicos ha aumentado exponencialmente la probabilidad de interferencia entre éstos si no se aplican criterios adecuados de diseño y fabricación. La idea sobre la que descansa la regulación de la CEM es la de proteger a los sistemas de comunicación, dispositivos o equipos cuyo funcionamiento pueda verse perjudicado por interferencias electromagnéticas, ya que pueden ocasionar tanto un riesgo en la seguridad de instalaciones y personas en caso de fallo del equipo, como un grave problema técnico y comercial para el fabricante. La compatibilidad electromagnética (CEM) es la aptitud de un equipo o sistema para funcionar satisfactoriamente en un ambiente electromagnético, sin introducir perturbaciones intolerables en ese ambiente o en otros equipos y soportar las producidas por otros equipos del mismo ambiente de trabajo.

\subsection{COMPATIBILIDAD ELECTROMAGNÉTICA EN LA UNIÓN EUROPEA}

El Mercado Único tiene uno de sus pilares fundamentales en la libre circulación de mercancías. Para lograr la plena consecución de esta circulación se han establecido tres mecanismos: armonización técnica, reconocimiento mutuo y evitar nuevas barreras al comercio.

La existencia de técnicas reglamentarias nacionales ha sido uno de los impedimentos para la creación de un Mercado Único. Se hacía necesaria una nueva reglamentación que estableciese los requisitos generales esenciales, redujese el control de las autoridades públicas previas a la comercialización de un producto e integrará la garantía de la calidad y otras técnicas modernas de evaluación de la conformidad.

La situación inicial está basada en la figura de la homologación, con tantos reglamentos técnicos como estados basados en normas técnicas de carácter obligatorio. El proceso de homologación se realizaba por tipo de producto por 
un organismo autorizado por cada Estado para ello. A todas luces no se trataba de un producto que favoreciera la competitividad de los productos (PORTILLO GARCÍA-PINTOS, 2005).

En un principio, desde la Unión Europea se pensó en una traslación de esta situación a nivel europeo, con la creación de reglamentos comunes para todos los Estados. Estos reglamentos tendrían forma de Directivas y se formularían por producto. El resultado de este proceso no fue el esperado, fracasando de nuevo por su excesiva rigidez. En I985 se acuerda mediante Resolución del Consejo plantear el Nuevo Enfoque de la Armonización y Normalización técnica, establece una nueva técnica y estrategia de reglamentación sobre la base de los siguientes principios:

- La armonización legislativa se limita a los requisitos esenciales que deben cumplir los productos comercializados en el mercado comunitario para poder circular libremente dentro de la Comunidad.

- Las especificaciones técnicas de los productos que cumplen los requisitos esenciales establecidos en las directivas se fijarán en normas armonizadas.

- La aplicación de normas armonizadas y de otro tipo seguirá siendo voluntaria y el fabricante siempre podrá aplicar otras especificaciones técnicas para cumplir los requisitos.

- Los productos fabricados en cumplimiento de las normas armonizadas gozan de la presunción de conformidad con los requisitos esenciales correspondientes.

En el ámbito de la Unión Europea, se entiende por normas armonizadas como aquellas normas europeas adoptadas por los organismos de normalización europeos, elaboradas de acuerdo con las Directrices Generales acordadas entre la Comisión y los organismos de normalización europeos y que siguen un mandato emitido por la Comisión, previa consulta a los Estado miembros.

En el sentido del Nuevo Enfoque se considera que existen normas armonizadas en cuando los organismos de normalización europeos presentan formalmente a la Comisión las normas europeas elaboradas o determinadas de conformidad con el mandato. La Legislación del Nuevo Enfoque se completó con la resolución del Consejo de ig89 en el que surge el llamado Enfoque Global en materia de evaluación de la conformidad. En ésta se fijan los principios para las distintas fases de la evaluación de conformidad: 
- Se introduce el enfoque modular.

- Se generan las normas europeas de garantía de calidad a cumplir por los productos (serie ISO 9000).

- Acuerdos de reconocimiento mutuo.

- Existencia de sistemas de acreditación.

La aportación principal del Enfoque Global es la de introducir un enfoque modular, que subdivide la evaluación de conformidad en varios etapas, módulos, en función de la fase de desarrollo de producto, el tipo de evaluación y la persona o entidad que realiza la evaluación.

El Enfoque Global fue completado por la Decisión del Consejo 90/683/ CEE, que a su vez fue sustituida y actualizada por la Decisión 93/465/CEE.; esta establece los módulos durante la fase de diseño y fabricación

\section{FIGURA I}

MÓDULOS MARCADO CE

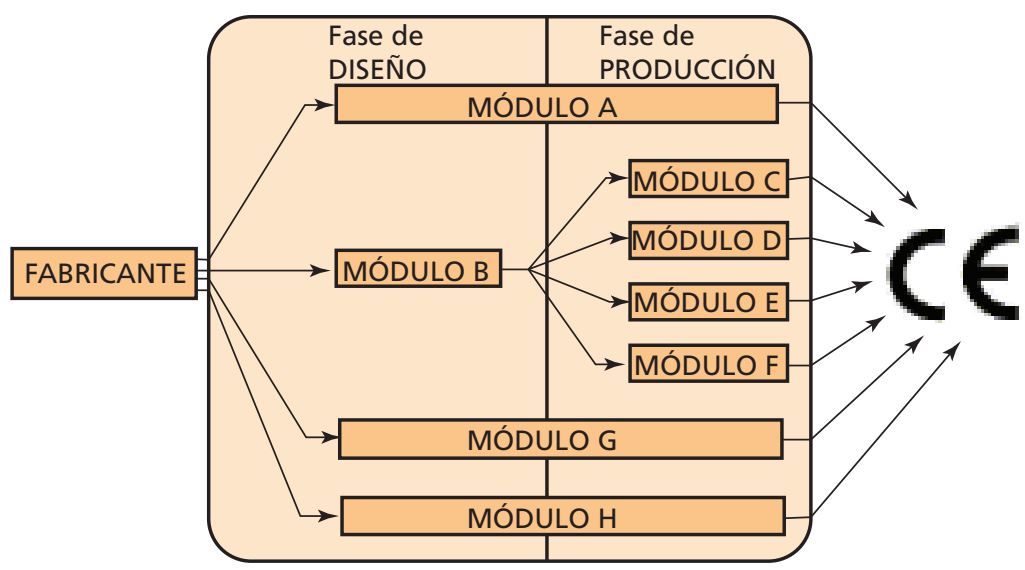

Fuente: www.ffii.nova.es/puntoinfomcyt.

- control interno de la fabricación;

- examen "CE" de tipo;

- conformidad con el tipo;

- aseguramiento de calidad de la producción;

- $\quad$ aseguramiento de calidad del producto;

- verificación de los productos;

- verificación por unidad;

- aseguramiento de calidad total. 
- LA RESPONSABILIDAD SOCIAL CORPORATIVA Y LA COMPATIBILIDAD ELETROMAGNÉTICA... • GREGORIO ESCALERA IZQUIERDO • CARMEN CALDERÓN PATIER

La característica más destacada de un producto una vez que ha obtenido el marcado "CE", es que el consumidor nunca deberá demostrar que el daño ha sido producido por el producto, sino que será obligación del fabricante o en su defecto el representante legal el que deberá demostrar que su producto no es el causante del daño.

El marcado CE simboliza la conformidad del producto con los requisitos establecidos por la Unión Europea al fabricante. En lo referente a la CEM, el marcado CE en los productos eléctricos y electrónicos constituye una declaración por parte de quien lo coloca que se cumplen las disposiciones comunitarias y que se han llevado a cabo los procedimientos de evaluación de conformidad pertinentes en materia de CEM. Podría darse el caso de que un producto estuviese sujeto a más directivas que las referidas a CEM, en ese caso la colocación del marcado CE supone el cumplimiento de todas y cada una de ellas. El marcado CE debe ser colocado por el fabricante o su representante autorizado establecido dentro de la Unión Europea.

\section{FIGURA 2}

\section{SÍMBOLO DEL MARCADO CE}

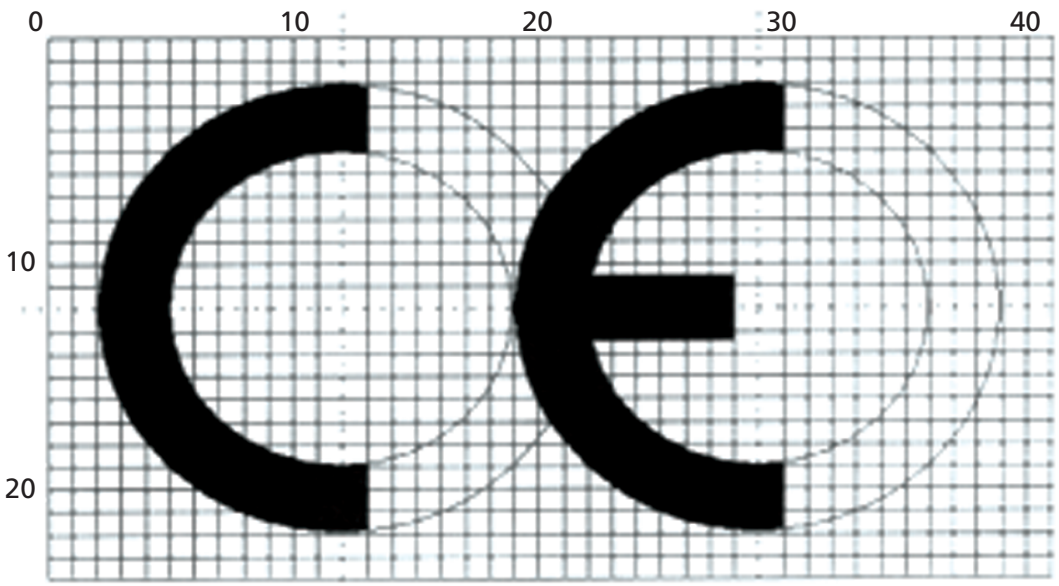

Fuente: Doce 93/465/CEE.

Los organismos notificados realizan las intervenciones correspondientes a las "terceras partes" que señalan los procedimientos de evaluación de la 
conformidad correspondientes a las directivas de Nuevo Enfoque que apliquen. Se trata de organismos que reúnen las competencias técnicas necesarias y que hayan sido notificados por los Estados miembros.

Las responsabilidades de estos organismos son:

- Proporcionar los servicios de evaluación de conformidad de acuerdo a las Directivas.

- $\quad$ Actuar imparcialmente, ser independiente de sus clientes.

- Proporcionar información pertinente a las autoridades, vigilancia del mercado, otros Organismos Notificados.

- Asegurar la confidencialidad de la información.

- Participar o estar representado en la normalización europea.

Podemos que la RSC en la Unión Europea está presente en las empresas de forma directa en cuanto su enfoque modular a la hora de fabricar los productos y de forma indirecta por los organismos notificados; los módulos están basados en la intervención de una primera parte, el fabricante, o de una tercera parte, organismo notificado.

Finalmente y teniendo como base la Propuesta de Directiva el I5 de diciembre de 2004 se aprueba la Directiva 2004/I08/CE relativa la aproximación de las legislaciones de los Estados miembros en materia de compatibilidad electromagnética y por la que se deroga la Directiva 89/336/CEE. Aunque esta Directiva se ha aprobado a finales de 2004 se ha instaurado un periodo hasta su entrada en vigor el 20 de julio de 2007 . Hasta esa fecha seguirá en vigor la Directiva 89/336/CEE.

\subsection{COMPATIBILIDAD ELECTROMAGNÉTICA EN ESTADOS UNIDOS}

En Estados Unidos el sistema de conformidad de productos es un sistema voluntario, con una intervención gubernamental prácticamente inexistente. El mercado es el principal regulador, de modo que la aceptación delos productos actúa como principal dinamizador a la hora de conseguir las distintas certificaciones.

Las normas de certificación las elabora principalmente el sector privado y las organizaciones de certificación son privadas y carecen de vinculación con autoridades gubernamentales. Existen varias entidades de reglamentación en función del sector implicado, en el caso de la CEM la principal entidad 
de reglamentación es la Comisión Federal de Comunicaciones (Federal Communications Comisión), en adelante FCC. Además pueden existir disposiciones adicionales generadas por diversas organizaciones a nivel federal, estatal y local.

Cada fabricante o importador deberá ser autorizado bajo una Declaración de Conformidad, o por la autorización de la Comisión por uno de los siguientes procedimientos: certificación o registro. Los siguientes párrafos definen los distintos procedimientos descritos en la 47 CFR Subpart J:

- Verificación. Es el procedimiento mediante el cual el fabricante efectúa las medidas o realiza las acciones necesarias para garantizar que sus equipos cumplen la normativa apropiada.

- Declaración de Conformidad. Es el procedimiento mediante el cual el Organismo Notificado hace las medidas o realiza las acciones necesarias para garantizar que se cumple la normativa apropiada. Estos organismos se encargan de enviar muestras a la FCC para demostrar que no es necesaria su certificación.

- Certificación. Consiste en la autorización por parte de la FCC basada en muestras suministradas por el fabricante.

Todas las regulaciones se encuentran incorporadas a la legislación de Estados Unidos mediante el Code of Federal Regulations. Sin embargo no existe un organismo coordinador centralizado del gobierno

La Compatibilidad Electromagnética dentro de los Estados Unidos está regulada, desde I975, por las Normas y Regulaciones FCC, título 47, parte I5, subapartado B. Se distinguen en esta norma dos categorías en función del ámbito de uso de los equipos:

a) Dispositivos Clase A. Con este marcado se distinguirían los utilizados en el ámbito comercial, industrial o de negocios.

b) Dispositivos Clase B. Este marcado se reserva a los equipos domésticos.

Los requisitos para los dispositivos de Clase A son menos restrictivos que los de la Clase B. Además los procedimientos administrativos para la verificación son más rigurosos en la Clase reservada a los equipos domésticos.

Las normas de la FCC que limitan las perturbaciones generadas por el equipo eléctrico o electrónico son prácticamente idénticas a las del CISPR (International Special Committee on Radio Inference) Pub. 22. La FCC estuvo de acuerdo en aceptar 
las medidas impuestas a los equipos electrónicos al demostrar cumplimiento con sus propias normas o con las especificaciones del CISPR-22 con pequeñas modificaciones. Esto significa que un equipo que cumple las normas CISPR cumple la reglamentación en materia de CEM. Por otra parte, las normas europeas CEM también tienen como base las especificaciones CISPR. Aunque no debe pensarse que la norma 47 CFR 15 Subpart B es totalmente equivalente a las Directivas Europeas de CEM tratadas en apartados anteriores. La norma americana sólo se refiere a equipos con emisiones en frecuencias radio.

En 1993 la FCC armonizó sus normas con el estándar CISPR 22. Como consecuencia, los vendedores pueden utilizar los límites de emisión definidos en la norma FCC Subpart B o en CISPR 22 para cualquier propuesta de verificación, certificación, notificación o Declaración de Conformidad.

La Declaración de Conformidad debe ser emitida por un laboratorio de pruebas autorizado, situado en un país reconocido por la FCC. Es el caso de los ordenadores personales y sus periféricos para los cuales se debe utilizar una etiqueta distinta en función de si se han realizado pruebas del sistema en conjunto, Figura 3, o si se ha ensamblado utilizando componentes certificados, Figura 4 .

Existe una vasta red de organismos de inspección y ensayos y autoridades con capacidad para llevar a cabo el trabajo requerido por las empresas y el gobierno. En general, estos organismos son de propiedad privada, en el caso de la FCC se utilizan laboratorios del sector privado para realizar pruebas de conformidad requeridas para recibir la autorización de equipos necesaria para su comercialización, fabricación o importación. La FCC realiza verificaciones esporádicas, antes y después de emitir las autorizaciones de equipos.

El sistema de certificación de productos, ampliamente utilizado, es de tipo voluntario y va más allá de las inspecciones al fabricante. Este sistema incluye una declaración de conformidad del proveedor, programas de verificación de asociaciones industriales y sindicatos, inspecciones por entidades independientes y programas donde se somete a los equipos a diversos ensayos y pruebas. En todos estos procesos no existe participación alguna de funcionarios de la Administración.

La FCC no acredita a los laboratorios para realizar verificaciones de la norma 47 CFR 15 Subpart B, no obstante, los laboratorios deben registrar la información en la FCC para demostrar que tienen la capacidad de realizar las pruebas necesarias. Las actuaciones de los distintos organismos varían en función del sector, desde las que no acreditan laboratorios pero los inspeccionan, como la FDA (Food and Drugs Administration.) o las que acreditan listados de laboratorios, como la EPA (Enviromental Proteccion Agency). 


\section{FIGURA 3}

\section{ETIQUETA DOC EQUIPO}

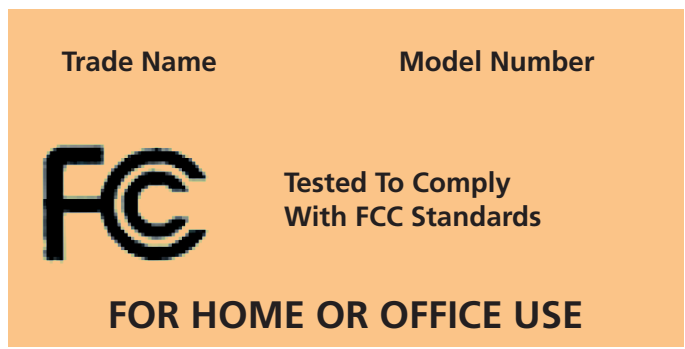

Fuente: Elaboración propia.

\section{FIgURA 4}

\section{ETIQUETA DOC ENSAMBLADO}

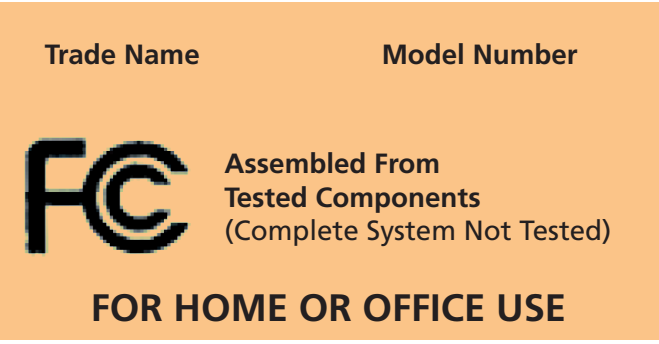

Fuente: Elaboración propia.

\subsection{COMPATIBILIDAD ELECTROMAGNÉTICA EN JAPÓN}

En Japón el sistema de conformidad de productos depende del sector, existiendo diferente legislación en función del área de mercado de los distintos productos. En los párrafos siguientes se describirán someramente los procesos de certificación en las áreas más significativas.

La Legislación relativa a la Seguridad y Salud Ocupacional (OSHA) en ambientes de riesgo o peligrosos, en vigor desde 2004 , regula la venta y uso 
de equipos eléctricos que tengan riesgo de explosión en entornos de trabajo como talleres o fábricas. La ley garantiza la seguridad y salud de trabajadores y la creación de un ambiente de trabajo confortable. La certificación TIIS (Technology Institution of Industrial Safety) es la necesaria para poder fabricar, comercializar o importar equipos industriales.

TIIS es una organización sin ánimo de lucro designada por el Ministerio de Trabajo desde I972 para certificar equipos en lo referente a la seguridad laboral. Hasta 2003 TIIS era la única organización con competencia para cerificar equipos en su ámbito de referencia. A partir de esta fecha, con la entrada en vigor de la Ley de Seguridad y Salud se ha modificado el procedimiento de certificación de modo que existen organismos registrados para la Certificación correspondiente a la Ley.

En cuanto a la Legislación para empresas de Telecomunicaciones la agencia JATE (Japan Approvals Institute for Telecommunications Equipment), es la designada por el Ministerio de Administración Pública, de Asuntos Domésticos y de Correos y Telecomunicaciones (MPHPT), antiguo Ministerio de Correos y Telecomunicaciones para la conexión de equipos a las redes de telecomunicaciones. No obstante, una vez los equipos estén marcados con la Certificación JATE, no precisarán de ninguna inspección adicional.

En Japón cuando cualquier compañía que tenga intención de fabricar o importar equipos médicos, deberá obtener una licencia del Ministerio de Salud, Trabajo y Asistencia Social. La aprobación para cada producto se obtendrá después de un análisis de sus características y será emitida por el Ministerio para cada planta del país.

La ley DENAM, en vigor desde el I de abril de 200 I (sustituye a la ley DENTORI de I96I), es la ley de aplicación para la certificación CEM de productos eléctricos y electrónicos, en su mayor parte de uso doméstico. Esta ley rige los asuntos de seguridad eléctrica y compatibilidad electromagnética, y es administrada por el Ministerio de Economía, Comercio e Industria, METI. Se dividen los productos reglamentados en dos grupos:

a) Especificados (SP). Son aquellos productos que requieren ser certificados por un Organismo de Evaluación de Conformidad.

b) No Especificados (NSP). Son aquellos productos que requieren conformidad obligatoria pero no necesariamente a través del Organismo de Evaluación de Conformidad del METI.

Cabe concluir que no existe una descripción única de los procesos de certificación de la calidad en Japón. Aún así, la tendencia parece ir en el sentido 
de la certificación por Organismos Notificados para cada una de las áreas de interés. Es la empresa japonesa es la de mayor regulación en cuanto a la RSC y por tanto deja menores grados de actuación a la misma.

\section{FIGURA 5}

\section{DIAGRAMA MARCAS PSE}

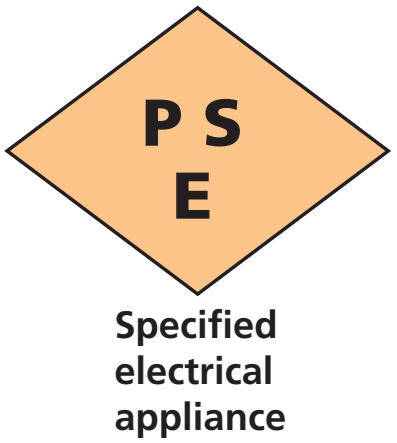

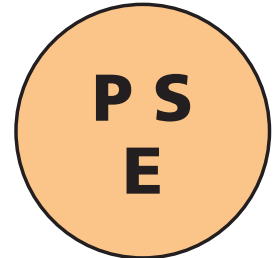

Non-specified electrical appliance

Fuente: DENAM, 200 I.

Se permite la autocertificación de equipos para el listado de equipos No Especificados. En cualquier caso los fabricantes e importadores de equipos eléctricos o electrónicos tienen el deber de notificar al METI, asegurar la conformidad con los estándares técnicos, conservar registros de las medidas y realizar un etiquetado correcto.

El Sistema de Certificación sólo se aplica a los productos listados en la Ley DENAM aunque la intención es la de extender la cobertura a todos los equipos del mercado. A modo de ejemplo citaremos que los ordenadores personales, a diferencia de las normas Europeas y Norteamericanas, no se encuentran dentro del listado y los fabricantes no están obligados a etiquetarlos excepto para aquellos componentes, como las fuentes de alimentación o cables, que sí están en el listado.

El Sistema de Certificación permite la utilización de estándares de la IEC y CISPR. Las certificaciones emitidas por terceras partes deben pertenecer al listado de Organismos Notificados por el MITE. Aquellos fabricantes sin oficina en Japón no podrán realizar notificaciones al METI por sí mismos sino por un organismo autorizado. 


\section{CONCLUSIONES}

El análisis delas diferentes normativas decertificación CEM en los tres grandes mercados occidentales, ponen claramente de manifiesto la falta de homogeneidad de las mismas y su clara relación con la interpretación (anglosajona, europea o asiática) de la ética empresarial o en los negocios, es decir de la RSC.

Así, mientras en el ámbito anglosajón prevalecen las normas del mercado como principal, y casi único, regulador, en el asiático, la regulación gubernamental es la predominante y por tanto con escaso margen de actuación de la RSC de las empresas, y por último Europa sería un nivel intermedio entre ambas.

Esta heterogeneidad en la interpretación y aplicación de la normativa de calidad que afecta directamente a los costes de los productos, hace que nos encontremos que el mercado europeo presenta mayores costes asociados a sus productos, haciéndolos menos competitivos en el mercado internacional.

Es de esperar que en una economía globalizada como la actual, y de hecho así se aprecia, una convergencia de procedimientos que abarate los costes al mínimo (como en Japón y USA) y será el propio mercado el que a medio plazo, se encargue de igualar las normas de calidad de los productos existentes como única vía de mantener la competitividad de las economías europeas. A continuación, en el Cuadro i se recoge un resumen de las analogías y diferencias esenciales en las tres áreas analizadas. 
- LA RESPONSABILIDAD SOCIAL CORPORATIVA Y LA COMPATIBILIDAD ELETROMAGNÉTICA...

\section{CUADro I}

\section{ANÁLISIS COMPARATIVO DE CRITERIOS}

DE CERTIFICACIÓN DE CALIDAD

\begin{tabular}{|c|c|c|c|}
\hline & JAPÓN & EUROPA & ESTADOS UNIDOS \\
\hline Regulación & $\begin{array}{l}\text { La empresa japonesa } \\
\text { es en materia de } \\
\text { certificación la } \\
\text { más regulada y } \\
\text { dependiendo del } \\
\text { producto ha de } \\
\text { ser certificado } \\
\text { por el ministerio } \\
\text { correspondiente, } \\
\text { por tanto deja poco } \\
\text { margen de acción a } \\
\text { la RSC. }\end{array}$ & $\begin{array}{l}\text { La empresa europea } \\
\text { su regulación no } \\
\text { es tan estricta y } \\
\text { permite de forma } \\
\text { directa mediante el } \\
\text { enfoque modular } \\
\text { que elijan ellas la } \\
\text { forma de obtener } \\
\text { la certificación } \\
\text { dentro de los } \\
\text { distintos módulos } \\
\text { posibles, y de forma } \\
\text { indirecta mediante } \\
\text { los organismos } \\
\text { notificados. Por } \\
\text { consiguiente la RSC } \\
\text { tiene margen de } \\
\text { maniobra. }\end{array}$ & $\begin{array}{l}\text { En Estados Unidos el } \\
\text { sistema de conformidad de } \\
\text { productos es un sistema } \\
\text { voluntario, con una } \\
\text { intervención gubernamental } \\
\text { prácticamente inexistente. } \\
\text { El mercado es el principal } \\
\text { regulador, de modo que la } \\
\text { aceptación de los productos } \\
\text { actúa como principal } \\
\text { dinamizador a la hora } \\
\text { de conseguir las distintas } \\
\text { certificaciones Por tanto la } \\
\text { RSC y sus Códigos éticos son } \\
\text { los que prevalecen. }\end{array}$ \\
\hline Objetivo & $\begin{array}{l}\text { Cumplir los requisitos } \\
\text { establecidos en la ley. }\end{array}$ & $\begin{array}{l}\text { La libre circulación } \\
\text { de mercancías en } \\
\text { todo el territorio de } \\
\text { la Unión Europea, } \\
\text { así como que los } \\
\text { productos reúnan los } \\
\text { requisitos mínimos de } \\
\text { seguridad. }\end{array}$ & $\begin{array}{l}\text { Que los productos } \\
\text { dependiendo de si son de } \\
\text { uso doméstico o industrial, } \\
\text { cumplan las exigencias } \\
\text { determinadas por las leyes } \\
\text { y por los códigos éticos de } \\
\text { las asociaciones a las cuales } \\
\text { se asocian las empresas, } \\
\text { debido a que son muy pocos } \\
\text { productos los que necesitan } \\
\text { la autorización por parte de } \\
\text { la FCC. }\end{array}$ \\
\hline
\end{tabular}




\section{CuAdro i (CONCLUSIÓN)}

\section{ANALISIS COMPARATIVO DE CRITERIOS} DE CERTIFICACIÓN DE CALIDAD

\begin{tabular}{|c|c|c|c|}
\hline & JAPÓN & EUROPA & ESTADOS UNIDOS \\
\hline Costes & $\begin{array}{l}\text { Todos los productos } \\
\text { poseen unos costes } \\
\text { de certificación y por } \\
\text { tanto menores en } \\
\text { cuanto a asociar estos } \\
\text { a la RSC. }\end{array}$ & $\begin{array}{l}\text { Todos los productos } \\
\text { tienen que ser } \\
\text { certificados, } \\
\text { mediante los } \\
\text { módulos o por } \\
\text { organismos } \\
\text { acreditados o } \\
\text { por ambos., por } \\
\text { consiguiente los } \\
\text { costes son mayores } \\
\text { en Europa que en los } \\
\text { otros dos grandes } \\
\text { mercados. }\end{array}$ & $\begin{array}{l}\text { Menores costes debido a } \\
\text { que son pocos productos los } \\
\text { que necesitan la autorización } \\
\text { de organismos estatales., } \\
\text { por tanto menos trabas } \\
\text { administrativas y menores } \\
\text { costes asociados. }\end{array}$ \\
\hline Globalización & $\begin{array}{l}\text { Cualquier empresa } \\
\text { que comercialice los } \\
\text { productos a nivel } \\
\text { internacional debe } \\
\text { poseer los requisitos } \\
\text { exigidos en cada } \\
\text { mercado. }\end{array}$ & $\begin{array}{l}\text { Cualquier empresa } \\
\text { que comercialice los } \\
\text { productos a nivel } \\
\text { internacional debe } \\
\text { poseer los requisitos } \\
\text { exigidos en cada } \\
\text { mercado. }\end{array}$ & $\begin{array}{l}\text { Cualquier empresa que } \\
\text { comercialice los productos } \\
\text { a nivel internacional debe } \\
\text { poseer los requisitos exigidos } \\
\text { en cada mercado. }\end{array}$ \\
\hline
\end{tabular}

Fuente: Elaboración propia.

\section{REFERENCIAS}

DENAN: DENKI YOHIN ANZEN HO. Ley de Equipos Eléctricos y Seguridad de los Materiales aprobada en abril de 200I por el Ministerio de Economía, Comercio e Industria de Japón.

DENTORI: DENKI YOHIN TORISHIMARI HO. Ley de Equipos Eléctricos y Control de los Materiales en vigor desde I96I por el 200I por el Ministerio de Economía, Comercio e Industria de Japón.

DIRECTIVA 889/36/CEE DOCE Serie L, n. I39 del 23 de mayo de I989. DIRECTIVA 92/3i/CEE del Consejo de 28 de abril de I992. 
- LA RESPONSABILIDAD SOCIAL CORPORATIVA Y LA COMPATIBILIDAD ELETROMAGNÉTICA... • GREGORIO ESCALERA IZQUIERDO • CARMEN CALDERÓN PATIER

DIRECTIVA 93/68/CEE del Consejo de 22 de julio de I993.

DIRECTIVA 93/465/CEE del Consejo de 22 de julio, que establece los módulos de evaluación de conformidad.

DIRECTRIZ 47 CFR Subpart J 2.909 of Federal Communications Commission.

DIRECTRIZ 47 CFR Parte I5, Subparte B.

ELECTROMAGNETIC FIELDS, publicado por la Oficina Regional de la OMS para Europa en I999 en Local Authorities, health and environment briefing pamphlet series; 32.

ESCALERA IZQUIERDO, G.; PASCUAL FAURA, M. La normalización y certificación como ventaja competitiva para la empresa española. Boletín Económico BICE 2820, p. 3-I3, 2004.

GALLARDO VELÁZQUEZ, A..Nuevas formas de organización frente a la reestructuración productiva. Revista Gestión Y Estrategia, n. I5, p. 54-65, I994.

GUÍA PARA LA APLICACIÓN de Directivas basadas en el Nuevo Enfoque y Enfoque Global de septiembre de I999.

GUIDELINES ON THE APPLICATION of council directive 89/336/EEC of 3 may I989 on the approximation of the laws of the member states relating to Electromagnetic Compatibility.

MELERO GUILLÓ, A. M.; CALATRAVA ANDRÉS, A. La industria electrónica en la Unión Europea. Boletín Económico BICE 2651, p. 32-43, 2000.

NORMA IEC 60050 I6I.

PALLÁS-ARENY, R.; SILVA, F. Compatibilidad electromagnética: una visión alternativa. Mundo Electrónico, v. 256, Iss 3, p. 42-45, I995.

PORTILLO GARCÍA-PINTOS, J. La certificación en el contexto de la seguridad industrial: criterios interpretativos y elementos de desarrollo. Revista Seguridad, n. I57, p. 25-33, 2005.

PROPUESTA DE DIRECTIVA DEL PARLAMENTO EUROPEO y del Consejo relativa a la aproximación de legislaciones de los Estados Miembros en materia de Compatibilidad Electromagnética de 23 de diciembre de 2002. COM (2002) 759 final.

\section{TRAMITAÇÃO}

Recebido em 15/3/2006

Aprovado em 27/6/2006 




This paper may be copied, distributed, displayed, transmitted or adapted if provided, in a clear and explicit way, the name of the journal, the edition, the year and the pages on which the paper was originally published, but not suggesting that RAM endorses paper reuse. This licensing term should be made explicit in cases of reuse or distribution to third parties. It is not allowed the use for commercial purposes.

Este artigo pode ser copiado, distribuído, exibido, transmitido ou adaptado desde que citados, de forma clara e explícita, o nome da revista, a edição, o ano e as páginas nas quais o artigo foi publicado originalmente, mas sem sugerir que a RAM endosse a reutilização do artigo. Esse termo de licenciamento deve ser explicitado para os casos de reutilização ou distribuição para terceiros. Não é permitido o uso para fins comerciais. 